\title{
SELF-CONSISTENT NUMERICAL MODELLING OF PULSAR MAGNETOSPHERES
}

\author{
H. Herold, T. ERTL, B. Finkbeiner, and H. RUder \\ Theoretische Astrophysik, Universität Tübingen
}

\begin{abstract}
The magnetosphere of a rapidly rotating, strongiy magnetized neutron star with aligned magnetic and rotational axes (parallel rotator) is modelled numerically. Including the radiation of the particles accelerated to relativistic energies as an efficient damping mechanism, we obtain a quasi-stationary self-consistent solution to this classical problem. The numerical simulation, which was started from the well-known vacuum solution, yields a global magnetospheric structure that can be characterized by two regions of oppositely charged particles, which eventually produce a relativistic pulsar wind, separated by a vacuum gap of considerable extent.
\end{abstract}

\section{Introduction}

The problem-fundamental to the physics of pulsars-of determining the global structure of the magnetosphere of a rapidly rotating strongly magnetized neutron star has not yet been solved selfconsisteatly (for a review cf. Michel 1982), but it must be solved before any conclusive interpretation can be given to the detailed body of observational data on pulsar radiation [cf. Taylor and Stinebring 1986, and references therein, as well as these Proceedings]. Apart from this reason, it is "... just intolerable that we should not know how a rotating magnetized neutron star comes to terms with its environment" (Mestel 1981).

We concentrate here on the self-consistent modelling of the magnetosphere of an aligned rotator, where the rotation axis is parallel to the magnetic axis of the neutron star. In this case, the homopolar induction, which should be responsible for populating the magnetosphere with charged particles pulled out from the neutron star surface via field emission (Goldreich and Julian 1969), can be studied in purity, whereas electromagnetic wave effects are neglected. The main questions to be answered are the following: What is the global structure of the electric and the magnetic field? How are the charge and current density distributed that produce these fields (together with the star)? Which particle populations exist in the magnetosphere?

\section{Basic equations}

\section{Maxwell and Vlasov Equations}

In the stationary axially symmetric case the elec. tromagnetic fields $E$ and $B$ in the pulsar magnetosphere can be described (in cylindrical coordinates $\rho, \varphi, z)$ by the electrostatic potential $\Phi(\rho, z)$, the magnetic flux function $\Psi(\rho, z)$, which determines the poloidal magnetic field, and the toroidal magnetic field $B_{\varphi}(\rho, z): \quad \boldsymbol{E}=-\nabla \Phi$ and $\boldsymbol{B}=$ $(1 / \rho) \nabla \Psi \times e_{\varphi}+B_{\varphi} e_{\varphi}$. The charge density $\rho_{e}$ and the current density $j$ determine the electric potential via the Poisson equation and the magnetic field via Ampere's law, which is here read in suitable units such that all quantities are dimensionless:

$$
\begin{gathered}
\Delta \Phi=-\rho_{\mathrm{e}} ; \quad\left(\Delta-\frac{2}{\rho} \frac{\partial}{\partial \rho}\right) \Psi=-\rho j_{\varphi} \\
\frac{\partial}{\partial z}\left(\rho B_{\varphi}\right)=-\rho j_{\rho} ; \quad \frac{\partial}{\partial \rho}\left(\rho B_{\varphi}\right)=\rho j_{z}
\end{gathered}
$$

The magnetosphere is formed by a collisionless plasma, in which the particles are expected to be extremely relativistic due to the huge electric and magnetic fields (the homopolar induction voltage between pole and equator of the neutron star is typically of the order $10^{17}-10^{18} \mathrm{~V}$ ). The charge and current densities are derived from the zeroth and first moments of the particle distribution function (for the sake of clarity, summing over the particle species has been omitted here)

$$
\rho_{\mathrm{e}}=\int f(\boldsymbol{r}, \boldsymbol{p}) d^{3} p ; \quad j=\int \boldsymbol{v} f(\boldsymbol{r}, \boldsymbol{p}) d^{3} p,
$$

where the distribution function $f(\boldsymbol{r}, \boldsymbol{p})$ is determined by the Vlasov equation

$$
\boldsymbol{v} \frac{\partial f}{\partial \boldsymbol{r}}+\frac{\partial}{\partial \boldsymbol{p}}[(\boldsymbol{E}+\boldsymbol{v} \times \boldsymbol{B}+\underset{\text { damping }}{\text { radiation }}) f]=0 .
$$

The velocity $v$ is given by $v=p / \sqrt{\left(\varepsilon^{2}+p^{2}\right)}$, where the dimensionless parameter $\varepsilon$ is defined by

$$
\varepsilon=2 m c^{2} / e B_{0} a^{2} \Omega,
$$

i.e. by the ratio between rest mass energy and homopolar induction energy $\left(B_{0}\right.$ is the polar magnetic 
field strength, $a$ the radius, and $\Omega$ the angular velocity of the neutron star). Because typical values for $\varepsilon$ are extremely small $\left(\varepsilon \sim-10^{-12}\right.$ for electrons, $\varepsilon \sim 10^{-\theta}$ for protons), the particles are expected to become extremely relativistic, since their maximum Lorentz factors should be $\sim|\varepsilon|^{-1}$, at least if the radiation reaction during phases of acceleration can be neglected. Although this is not the case, as will be discussed in the following, it is nevertheless convenient to use a scaled Lorentz factor $\Gamma=\varepsilon \gamma$ that never exceeds unity.

\section{Particle motion in the limit of strong radiation damping}

Studying the trajectories of particles in realistic pulsar fields, one can get an idea of how strongly the radiation reaction does affect the particle motion.

The Lorentz-Dirac equation of motion in the Landau approximation ( $c f$. Landau and Lifshitz 1975) can be written (for $|\varepsilon| \ll 1)$ as

$$
\dot{\boldsymbol{v}}=\frac{1}{\Gamma} \boldsymbol{F} ; \quad \dot{\Gamma}=\boldsymbol{E} \cdot \boldsymbol{v}-D_{0} \Gamma^{2} \boldsymbol{F}^{2}
$$

with

$$
F=E+v \times B-v(E \cdot v)
$$

The dimensionless damping constant

$$
D_{0}=\frac{2}{3} \frac{e^{2}}{4 \pi \epsilon_{0}} \frac{\Omega}{m c^{3}} \frac{1}{\varepsilon^{3}},
$$

characterizes the strength of the radiation reaction. For typical pulsar parameters, its value is of the order of $-10^{14}$ for electrons, and 10 for protons. Thus, at least for the electrons, the radiation reaction force dominates the particle motion. Large values of $\left|D_{0}\right|$ imply that the factor of $D_{0}$ in eq. (5a) always remains very small; this leads us to the assumption, which was confirmed by numerical integration of eqs.(5) [cf. Herold et al. (1985), Herold et al. (1986)], that during the motion the condition $F \approx 0$ is fulfilled, which means that the radiation reaction is locally minimized along the trajectory. This is a condition for the velocity and yields, for given $E$ and $B$ fields

$$
\begin{aligned}
v & =\frac{1}{B^{2}+P^{2}}\left[E \times B+\frac{1}{P}(E \cdot B) B+P E\right](7 a) \\
P^{2} & =\frac{1}{2}\left(E^{2}-B^{2}\right)+\frac{1}{2}\left[\left(E^{2}-B^{2}\right)^{2}+4(E \cdot B)^{2}\right]^{\frac{1}{2}}
\end{aligned}
$$

Thereby, we have obtained a local velocity field $v=v(E, B)$ and thus a fluid-like picture for the particle motions in the magnetosphere. The main characteristics of this "damping-free" motion is that the particles try to come to rest in surfaces where
$\boldsymbol{E} \cdot \boldsymbol{B}=0$. How such strong radiation reaction effects influence the particle motions in the case of an oblique rotator, has been discussed recently by Finkbeiner et al. (1989).

\section{Numerical approach and results}

\section{Numerical method}

Based on these results, the task of determining a self-consistent solution is simpler than before, but still difficult due to the great non-linearity of the problem. Our approach is based on the idea of starting from the vacuum solution and then filling up the magnetosphere with the particles that are pulled out from the neutron-star surface. This is not a real time-dependent calculation, since we assume that the electric field is always described by an electrostatic potential, but for transporting the charge with the velocity of eq.(7) we must solve the time-dependent continuity equation

$$
\dot{\rho}_{\mathrm{e}}+\operatorname{div}\left(\rho_{\mathrm{e}} \boldsymbol{v}\right)=0,
$$

and therefore $\dot{\boldsymbol{E}}$ cannot be omitted in Ampere's law. Thus eqs. (1b) have to be replaced by

$$
\left(\Delta-\frac{2}{\rho} \frac{\partial}{\partial \rho}\right)\left(\rho B_{\varphi}\right)=\rho\left(\frac{\partial j_{z}}{\partial \rho}-\frac{\partial j_{\rho}}{\partial z}\right) .
$$

(Actually, the continuity equation is solved for each particle species as the velocity of eq. (7a) depends on the particle charge: the sign of the quantity $P$ $(=\boldsymbol{E} \cdot \boldsymbol{v})$ from eq. $(7 \mathrm{~b})$ has to be identical to that of the charge. Thie charge and current density are calculated by summation over both species.]

In summary, we solve at each time step the elliptic equations (1a) with Dirichlet boundary conditions for $\Phi$ and $\Psi$ on the star-we assume a ho mogeneously magnetized neutron star(the change of $\Phi$ at infinity has to be derived from Ampere's law)-and eq.(9) with the Neumann boundary condition $\partial\left(\rho B_{\varphi}\right) / \partial r=-\rho j_{\vartheta}$ on the star's surface, where for the outcoming current the simple emission law $j=\sigma E_{\|}$is assumed, with a constant value of $\sigma$. The three elliptic equations are solved by successive over-relaxation (SOR) in a vectorizable checkerboard scheme. For the continuity equations (8) an explicit discretization in time with 2dimensional Flux Corrected Transport is used in order to preserve steep gradients in the charge density. A more detailed description of our numerical methods is given by Erti (1988).

\section{Results}

The results described below were obtained for a rapid rotator with $r_{0}=(\Omega a) / c=0.1$ as the ra- 


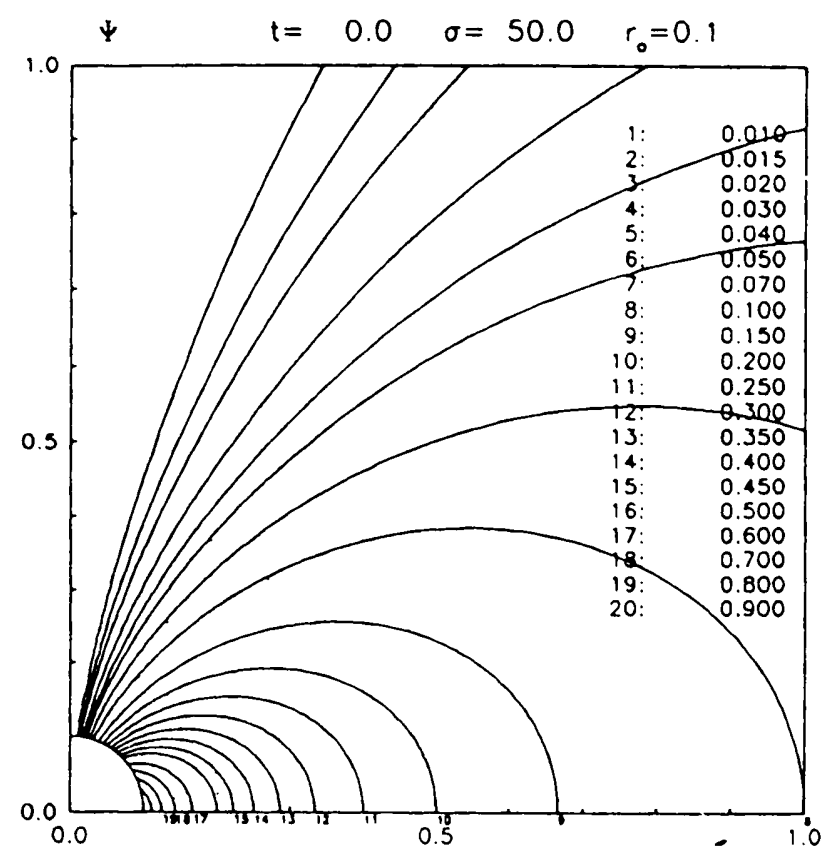

Figure 1 Initial phase: contour lines of the flux function $\Psi$, i.e. magnetic field lines for a dipole configuration.

tio of stellar and light cylinder radii (Ertl et al. 1987, Herold et al. 1989). We start with the vacuum fields of an uncharged aligned rotator, i.e. an electric quadrupole and magnetic dipole field resulting in $\boldsymbol{E} \cdot \boldsymbol{B} \leq 0$ everywhere. For comparison with later results, we present, in figures 1 and 2 the initial configuration of the magnetic and electric fields.

Thus, as long as $\boldsymbol{E} \cdot \boldsymbol{B} \leq \boldsymbol{0}$ at the surface, only negative particles can be emitted and then transported along the magnetic field lines towards the equator where they accumulate. This causes a change in the electric field such that the $E \cdot B=0$ surface rises from the equator towards the polar field line, forcing the negative particles to follow it. As soon as $E \cdot B>0$ at some part of the star's surface, positive particles can enter the magnetosphere.

Then a steadily increasing cloud of positive particles develops in the equatorial region near the star, whereas the negative particle distribution evolves more and more into a cone-like structure above the magnetic pole. During this phase the electric field changes its structure considerably. After about one revolution the magnetosphere has developed a structure which already resembles the final situation, although one has to follow the evolution for a longer time in order to come close to stationarity. (As indicators for time-independence we use various global quantities like the total charge of the star and the total charges of negative or positive particles in the magnetosphere as well as the total particle currents out of the star and at infinity.) This quasi-stationary end phase is depicted (after $30 \mathrm{rev}$ olutions; one revolution of the star corresponds to

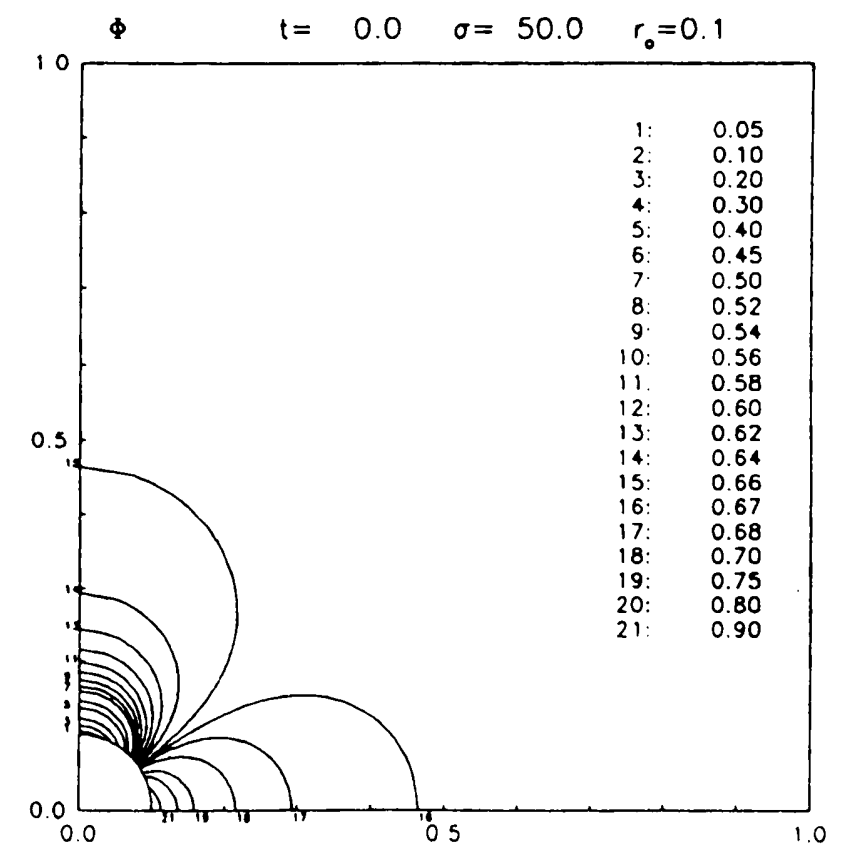

Figure 2 Initial phase: contour lines of the electrostatic potential $\Phi$, depicting the quadrupole electric field.

$t=2 \pi$ ) in figures 3-6, where the charge densities and contour lines both of the electrostatic potential $\Phi$ and of $E \cdot B$ are shown.

Two charged-particle regions (the negative ones in a cone around the polar field line, the positive ones around the equatorial plane) separated by a vacuum gap are seen. [Vacuum gaps in non-selfconsistent models are not new of. Mestel et al. (1985).] In this vacuum gap $E_{\|}=(\boldsymbol{E} \cdot \boldsymbol{B}) /|\boldsymbol{B}|$ is greatly different from zero ( $c f$. figure 6 ), whereas in regions populated with plasma the electric field (cf. figure 5)-now utterly dissimilar to the initial quadrupole (cf. figure 2)-has evolved as to achieve $\boldsymbol{E} \cdot \boldsymbol{B} \sim 0$ there. The poloidal magnetic field does not change very much; it remains essentially dipolar and is practically identical to that in figure 1. The neutron star is now positively charged, and the positive and the negative currents out of the star exactly match each other.

From the velocity fields (not shown) one can conclude that the positive particles corotate with the star (to a very good approximation) as long as they are inside the light cylinder, but close to the light cylinder they cease to corotate and stream as through a nozzle into the outer magnetosphere forming a radial wind. The velocity of the negative particles is nearly poloidal and thus this wind builds up at greater distances from the star.

There are some wave-like fluctuations, especially inside the negative zone, which seem to originate from the tendency of the system to make $E \cdot B$ small (or even zero) in the plasma regions. This explains the strange folded structure of the $E \cdot B=0$ surface and might be an indication that the mag- 


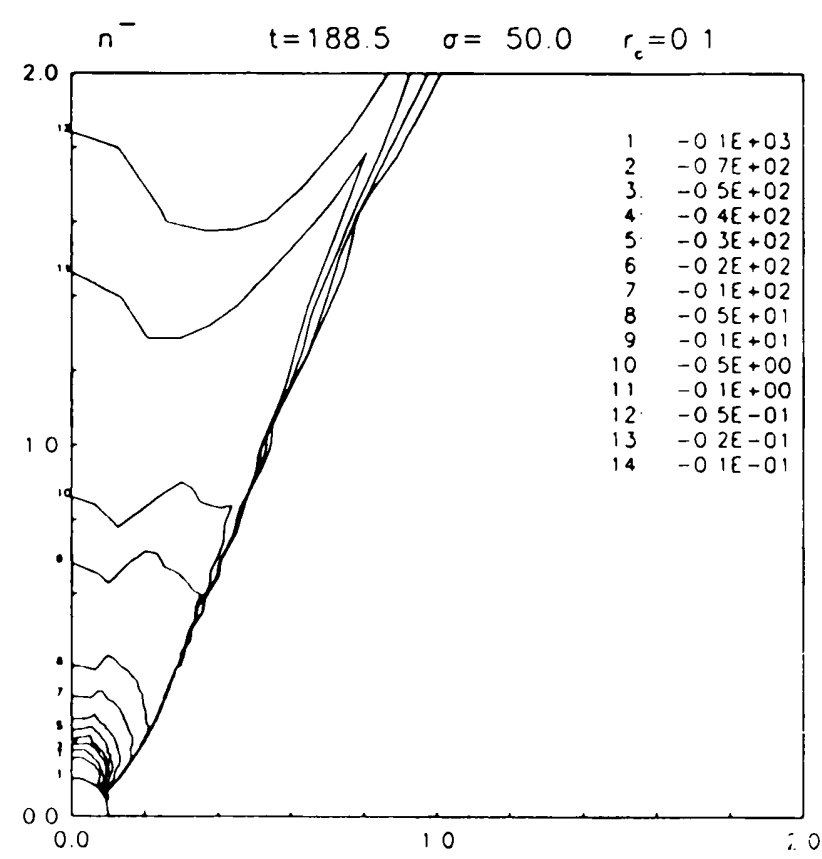

Figure 3 Quasi-stationary end phase: contour lines of the density of negative particles.

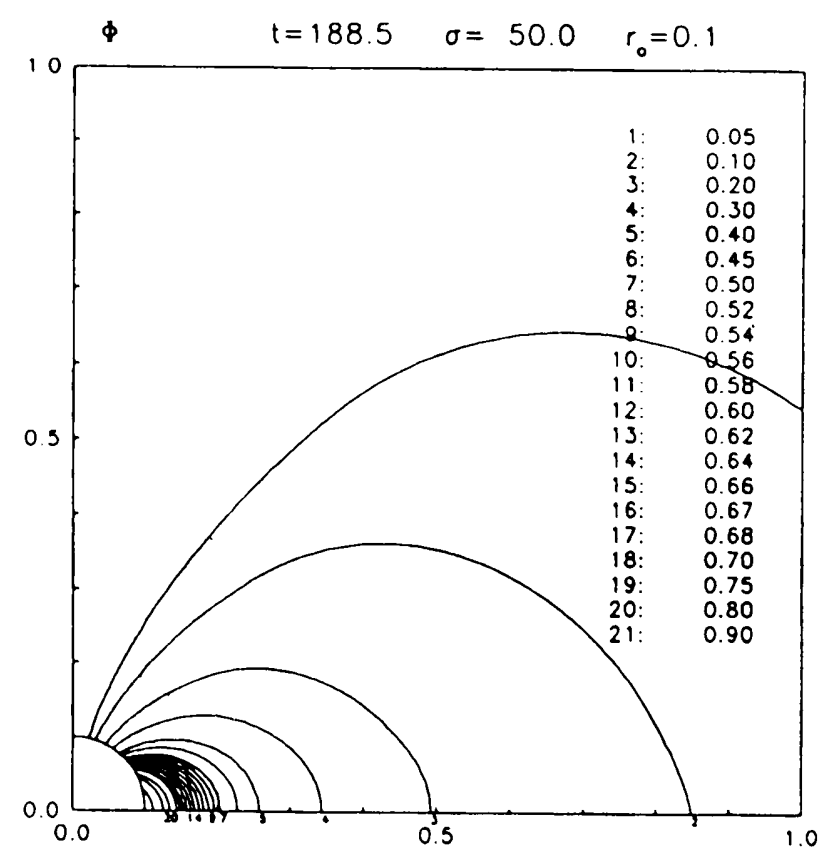

Figure 5 Quasi-stationary end phase: contour lines of the electrostatic potential $\Phi$. The contour values are the same as in figure 2.

netosphere wants to form an extended region with $E \cdot B=0$; however, this is difficult to achieve with a relativistic particle population. Whether for this end a cool non-relativistic plasma population is necessary or whether such fluctuations are a physically real part of a quasi-stationary configuration is an open question. Globally, our solution represents a stationary structure; for instance, the charges of the star and of the magnetosphere remain, in the end phase, constant with great accuracy.

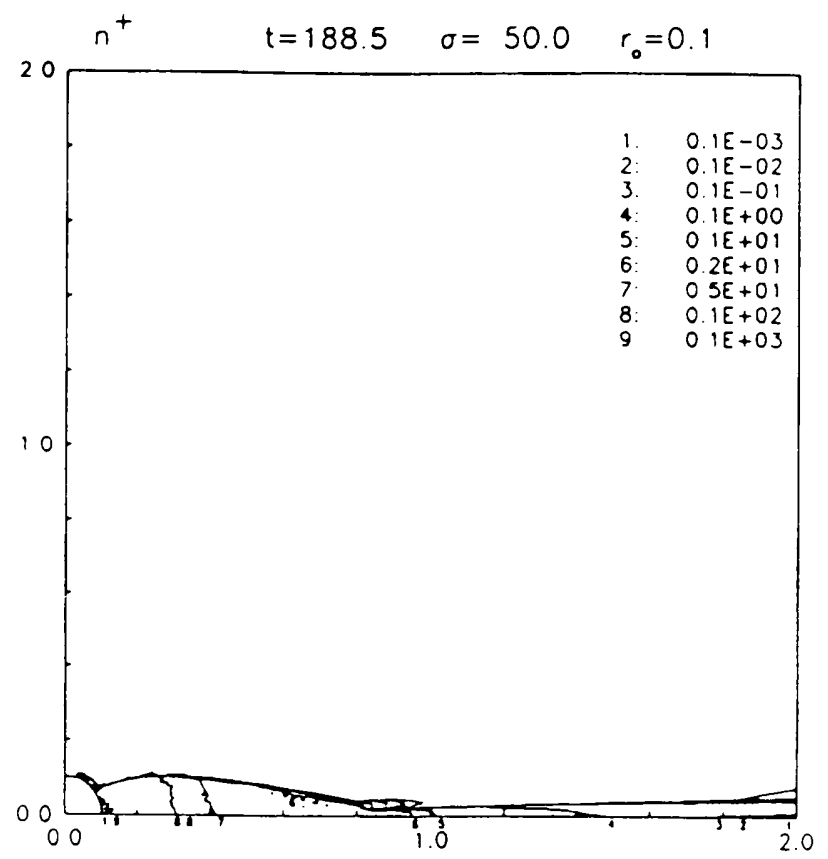

Figure 4 Quasi-stationary end phase: contour lines of the density of positive particles.

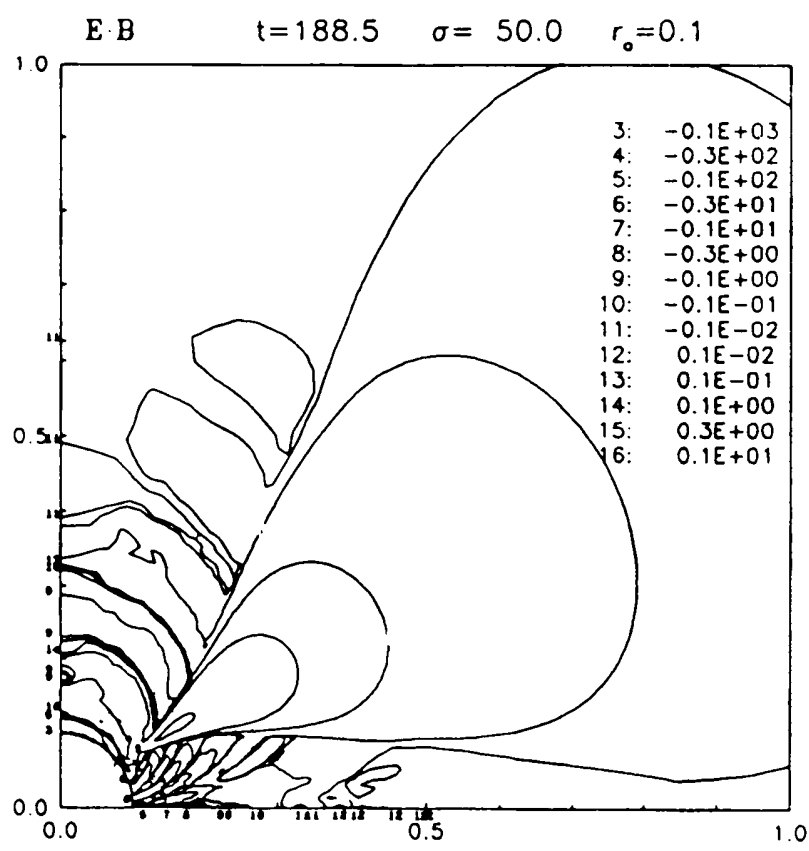

Figure 6 Quasi-stationary end phase: contour lines of $E \cdot B$. In the plasma regions the parallel electric field tends to become small.

For the first time in pulsar magnetospheric theory it seems that a stationary self-consistent solution for the aligned rotator has been found by a quasi-time-dependent simulation. Detailed diagnostic investigations of this solution, such as the calculation of the radiation of the accelerated particles, as well as studies of the parameter dependencies (rotation frequency $\Omega$, and emissivity $\sigma$ of the neutron star) are tasks for the future. 